\title{
A CASE OF SEPTIC SHOCK WHICH POSTMORTEM PATHOLOGICAL AUTOPSY REVEALED BACTERIAL MYOCARDIAL ABSCESS
}

National Hospital Organization Saitama National Hospital Tsunenori Tamai

\section{Background}

The myocardial abscess is a scarce condition, but it is significant due to its high mortality. We aimed to seek insight into it by presenting a case report.

Case

A 73 years old male was emergently transferred to our hospital with disorientation and hypotension. He had a past medical history of diabetes mellitus, hypertension, myocardial infarction, atrial fibrillation, and calculous cholecystitis. He had complained of lumbago since 1 week before arrival at our hospital.

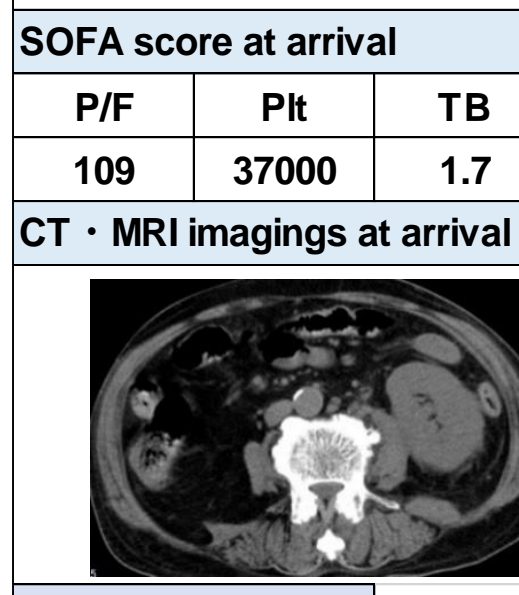

Clinical course

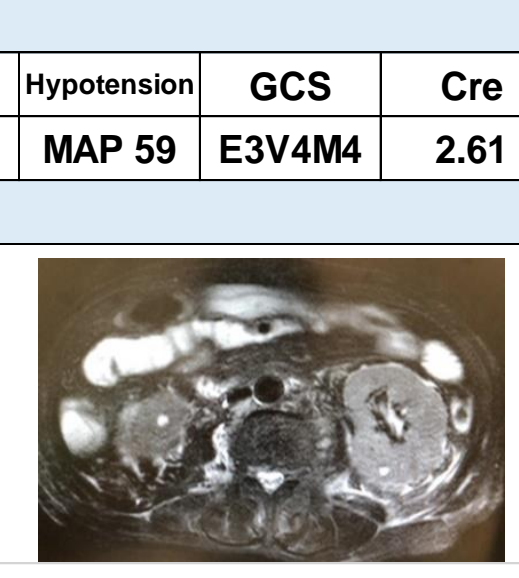

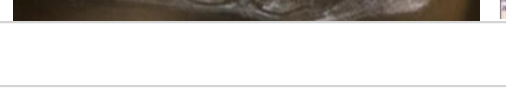

Oxygen therapy with non-rebreather mask

Recombinant human soluble thrombomodulin

MEPM + VCM

Noradrenaline

Fluid resucitation

mechanical ventilation

Total score

Blood culture

Staphylococcus aureus (MSSA) ECG after ROSC
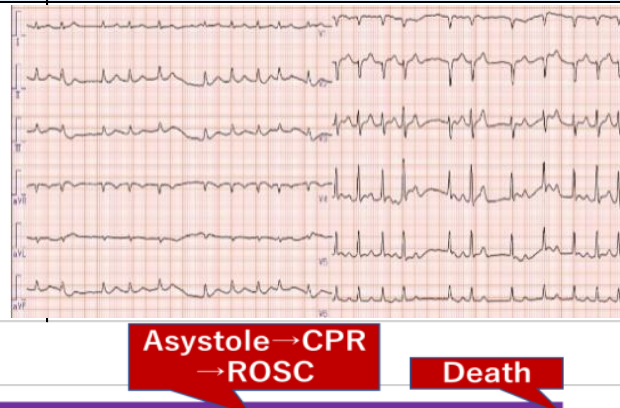

Major autopy findings

1.Left psoas abscesses with pyogenic spodylodiscitis

2.Myocardial abcesses with myocarditis

3.Small old infarctions in left ventricular septum and endocardium

4.Atherosclerosis of the left anterior descending artery with $50 \%$ stenosis

5.Bilateral renal abcesses

Discussion

Prevalence of myocardial abscess

$0.2 \%$ to $1.5 \%$ in the autopsy cases

(1)

Three types of pathogenic features about the formation of myocardial abscesses

1.Myocardial abscesses in the setting of overwhelming sepsis and the presence of other organs with abscesses in the absence of endocarditis

2.Myocardial abscesses due to direct extension from bacterial endocarditis

3.Abscesses only in the myocardium and absent in other organs

(1)

Conclusion

Our case was due to overwhelming staphylococcal sepsis from pyogenic spondylitis. The vasoplegia due to the septic shock was too severe to survive for the patient until presenting the symptoms of myocardial abscesses. We might not be able to notice the presence of myocardial abscesses without the autopsy. 